\title{
Pesquisa com famílias: aspectos teórico-metodológicos
}

\author{
Elisângela Böing \\ Maria Aparecida Crepaldi \\ Carmen Leontina Ojeda Ocampo Moré \\ Universidade Federal de Santa Catarina, Florianópolis-SC, Brasil
}

\begin{abstract}
Resumo: Este artigo apresenta reflexões conceituais e teórico-metodológicas consideradas relevantes no planejamento de pesquisa com famílias. Evidenciam-se as consequiências da transição paradigmática da ciência na produção de conhecimento relacionada à família, principalmente, na variação dos objetos de estudo e estratégias de investigação. Frente à complexidade do tema família, para a qual converge uma multiplicidade de olhares teóricos possíveis, surge a necessidade do uso adequado e combinado de diferentes estratégias e instrumentos metodológicos. Entende-se que a compreensão da complexidade das famílias não pode ser atingida com um único estudo, mas sim por meio da conjunção de diversos estudos, apontando a importância e necessidade da contextualização do processo de investigação para melhor responder às demandas científicas e sociais.
\end{abstract}

Palavras-chave: Pesquisa científica. Família. Métodos de pesquisa. Psicologia.

\section{Research on families: theoretical and methodological aspects}

\begin{abstract}
This article presents conceptual and theoretical-methodological aspects considered important in the planning of research on families. The consequences of the pragmatic transition of science, in the production of knowledge related to the family, are evidenced, especially in the variation of objects of study and strategies of investigation. Considering the complexity of the topic "family", to which a multiplicity of theoretical perspective converges, emerges the need of adequate and synchronized use of different strategies and methodological instruments. Understanding of families' complexity cannot be achieved through a single study but a mix of several studies, which appoints to the importance and need of contextualizing the investigation process to better respond to scientific and social demands.
\end{abstract}

Keywords: Scientific research. Family. Research methods. Psychology.

\section{Estudios con familias: cuestiones teórico-metodológicas}

Resumen: El presente artículo presenta reflexiones conceptuales y teórico-metodologicas consideradas nucleares para el planeamiento de investigaciones con familias. Se analizan las consecuencias de la transición paradigmática de la ciencia en la producción del conocimiento vinculado a la familia, principalmente, en las variaciones de los focos de estudio y estrategias metodologicas de intervención. Reconociendo la dimensión de la complexidad inherente al tema familia, en el cual converge una multiplicidad de posicionamientos teóricos posibles, surge la necesidad de un uso adecuado y combinado de diferentes estrategias e instrumentos metodológicos. Se considera que la comprensión de la complexidad, en los estudios con familias, se da através de la conjunción de diversos estudios, destacando la importancia y necesidad de la contextualización del proceso de investigación, para tener una respuesta más coherente con las realidades científicas y sociales.

Palabras clave: Investigación cientifica. Familia. Metodologia de la investigación. Psicología 


\section{Introdução}

Considerando a importância do estudo com famílias para a ciência psicológica atual e o crescente interesse dos pesquisadores por esta temática, este artigo tem como objetivo abordar aspectos conceituais e teórico-metodológicos relevantes para o planejamento de pesquisa com famílias. Para contextualizar, epistemologicamente, como a família se tornou objeto de estudo da psicologia, cabe resgatar do curso da ciência, a transição paradigmática iniciada com a chamada "revolução científica". Neste sentido, um forte marco do avanço da ciência se deu nos séculos XVII e XVIII, com Descartes, Newton, John Locke e Adam Smith, fundadores do Iluminismo, movimento que, com idéias que derrubaram o Estado Absolutista, lançaram base do racionalismo e mecanicismo. Destacam-se como pressupostos epistemológicos desta ciência moderna (tradicional): a simplicidade, a estabilidade e a objetividade, merecendo uma breve contextualização para melhor evidenciar a concepção de realidade e sua relação com o investigador, assim como a proposta metodológica de abordar a mesma (Esteves de Vasconcellos, 2003, 2005).

O pressuposto da simplicidade remete à crença de que é preciso separar o objeto em partes para entender o todo, daí decorre a atitude de análise e a busca das relações causais lineares. O pressuposto da estabilidade segundo o qual o mundo é estável, que "já é como é". Ligados a esse pressuposto estão as crenças na determinação (com a conseqüente previsibilidade dos fenômenos) e na reversibilidade (com a conseqüente controlabilidade dos fenômenos). Além do pressuposto da objetividade, ou seja, a crença de que existe uma realidade independente do observador e que é possível e indispensável ser objetivo, como critério de cientificidade, na constituição do "conhecimento verdadeiro" do mundo, da realidade (Esteves de Vasconcellos, 2003, 2005).

A ciência moderna e seus pressupostos defrontam-se com uma crise profunda e irreversível, fruto da revolução científica iniciada com os físicos Max Plank, Einstein (com o conceito de relatividade), Niels Bohr, Boltzman, Heisenberg (com o princípio da incerteza, demonstrando a interferência do observador na observação/medição de um objeto). Frente às no- vas descobertas, as leis da física passaram a assumir caráter probabilístico e a hipótese de determinismo mecanicista é inviabilizada uma vez que a totalidade do real não se reduz à soma das partes, evidenciando-se, ainda, a complexidade da distinção sujeito/objeto (Aun, Esteves de Vasconcelos, \& Coelho, 2005; Esteves de Vasconcellos, 2003; Sousa Santos, 2006).

Recentemente, sobretudo nas três últimas décadas do século XX, somam-se as contribuições de diversos outros cientistas que através de conceitos e teorias se colocaram como condições da crise do paradigma dominante, dentre os quais se destacam: o químico russo Ilya Prigogine; o físico e ciberneticista austríaco Heins Von Foster; o biofísico francês Henri Atlan e os biólogos chilenos Humberto Maturana e Francisco Varela. Realizando pesquisas em conformidade com o paradigma tradicional da ciência, os cientistas encontraram resultados que lhes mostraram os limites desse paradigma. Viram irreversibilidade e incontrolabilidade em alguns processos físicos, como os fenômenos do calor; reações de sistemas físicoquímicos que operam longe do equilíbrio (quando as flutuações podem ser amplificadas e o sistema evolui através de saltos qualitativos) e determinação histórica no funcionamento de estruturas dissipativas químicas (Aun e cols., 2005; Esteves de Vasconcellos, 2003; Sousa Santos, 2006).

Estas teorias culminam em uma nova concepção de matéria e natureza e fazem parte de um movimento convergente, sobretudo a partir de meados dos anos setenta, que atravessa as várias ciências, inclusive as ciências sociais. Este movimento traz inovações teóricas que propiciam uma profunda reflexão epistemológica sobre o conhecimento científico. As leis assumem caráter probabilístico, aproximativo e provisório. Na biologia, em que as interações entre fenômenos e formas de auto-organização são mais visíveis, a noção de lei foi sendo substituída pelas noções de sistema, estrutura, modelo e processo. Os objetos passam a ter fronteiras cada vez menos definidas a tal ponto que os objetos em si são menos reais que as relações, em teias complexas, entre eles (Esteves de Vasconcellos, 2003; Morin, 1996; Sousa Santos, 2006). 
O pensamento sistêmico, proposto por Esteves de Vasconcellos (2003) como a "ciência novoparadigmática", apresenta mudanças em relação aos três pressupostos epistemológicos da ciência tradicional: do pressuposto da simplicidade para o pressuposto da complexidade, buscando a contextualização dos fenômenos e reconhecendo a causalidade recursiva; do pressuposto da estabilidade para o da instabilidade, entendendo que o mundo está em processo dinâmico de constantes transformações e a consequiente imprevisibilidade de alguns fenômenos, e do pressuposto da objetividade para o pressuposto da intersubjetividade, reconhecendo que não há uma realidade independente do observador, que o conhecimento científico é construção social, em espaços consensuais, por diferentes sujeitos/observadores.

Nesse sentido, uma das tarefas do pesquisador é compreender e explicitar o próprio papel, seus limites, possibilidades e implicações, contextualizando o seu fazer. Por sua vez, acerca do suporte teórico da "biologia do conhecer", Maturana e Varela (1995) mostraram que, mais do que a existência de múltiplos pontos de vista, cada um destes possui critérios de validade, já que é impossível acessar uma realidade objetiva que exista independentemente de sua coconstrução.

Segundo o pensamento sistêmico tudo no universo está interligado através de um emaranhamento de ações, interações e retroações. Este é o princípio básico da epistemologia da complexidade desenvolvida por Morin (1990, 1996). Segundo o autor, não se trata de um pensamento que "abre todas as portas", não é onisciente, pois sabe que sempre é local, situado em um tempo e momento, não é completo, pois sabe de antemão que sempre há incertezas. Assim, ao adotar o ponto de vista da complexidade, sabe-se que é impossível um ponto de vista onisciente e que o caminho possível é a construção de metapontos de vista - limitados e frágeis - requisito que diferencia o pensamento simples, que acredita em verdades, em realidade independente do observador, e o conhecimento complexo que aponta para a necessidade da "curva auto-observável" do observador-conceituador sobre si mesmo.
Observa-se que a produção de conhecimento, no processo de investigação da ciência moderna, sofre o impacto dos avanços, nos diferentes campos das disciplinas que a compunham, evidenciando por um lado, uma espécie de desconstrução das crenças presentes em seu paradigma e, por outro, o surgimento de novos pressupostos epistemológicos. Isto tornouse necessário para superar os limites evidentes da mesma, no sentido de poder responder assim, ao reconhecimento das realidades multifacetas de pesquisa, em que a contextualização se impunha para dar conta da complexidade dos fenômenos estudados.

Especificamente no campo da psicologia, estudos advindos do modelo tradicional de ciência trouxeram inovações teóricas que desencadearam uma profunda reflexão epistemológica sobre o conhecimento psicológico, com importantes implicações diretas na prática científica.

No modelo da ciência tradicional, dentro da perspectiva da objetividade, o universo psicológico, como qualquer outro, era concebido como passível de ser conhecido sem qualquer interferência do observador e do ato de observar (Grandesso, 2000).

Conforme essa epistemologia foi sendo questionada, novos modelos teóricos foram desenvolvidos. Os sistemas familiares, assim como os sistemas sociais, deixam de ser concebidos como estruturas mecânicas coisificadas e passam a ser compreendidos como sistemas intersubjetivos compostos por agentes conscientes, intencionais que se co-criam a si mesmos e a seu entorno em uma permanente interação comunicativa e construção de significados. Ocorre uma mudança de foco do indivíduo para os sistemas humanos, do intrapsíquico para o interrelacional (Goolishian \& Anderson, 1996; Grandesso, 2000).

Ao assumir um outro enfoque para compreender o comportamento humano, por considerar o indivíduo dentro de um contexto interacional, a atenção passa a ser voltada para a comunicação e o comportamento de todos os membros envolvidos no sistema, nos elos de recursividade entre os membros e aos contextos em que estão inseridos (Aun e cols., 2005; Grandesso, 2000). 
Visando a contextualização dos fenômenos, a família se mostra para a psicologia como um objeto de pesquisa precioso nos estudos sobre desenvolvimento humano, entendendo a família e a cultura como contextos essenciais para a compreensão do indivíduo em sua singularidade. Focalizar a singularidade e a complexidade da rede relacional familiar permite vislumbrar a família como um grupo específico em desenvolvimento, inserido em um contexto cultural também em desenvolvimento (Dessen \& Silva Neto, 2000).

\section{O enfoque sistêmico como possibilidade de abordagem teórica na pesquisa com famílias}

A respeito da transição paradigmática da ciência e das implicações dessa na prática científica, muitos autores apontam que se trata de uma transição difícil e lenta e ressaltam a dificuldade da incorporação efetiva de um novo paradigma. Nesse sentido, afirmam que a reflexão epistemológica se mostra muito mais avançada e sofisticada que as práticas científicas, evidenciando, assim, uma fase de transição e insegurança (Cecchin, 1996; Esteves de Vasconcellos, 2003; Morin, 1996; Sousa Santos, 2006).

Esta fase de transição pode ser visualizada no universo acadêmico onde, apesar da forte presença da ciência tradicional na prática científica, muitos pesquisadores compartilham a respeito da impossibilidade de, através de um estudo, se capturar uma realidade objetiva, entendendo que não existe uma realidade "de fato", mas representações de realidades que são influenciadas por fatores, tais como: abordagem teórica e metodologia adotadas no estudo e dados da biografia do próprio pesquisador, como: vivências; interesses, conhecimento, representações (Biasoli-Alves, 1998; Cecconello \& Koller, 2004; Dessen \& Murta, 1997; Dessen \& Silva Neto, 2000; Esteves de Vasconcellos, 2003; Flick, 2004).

A epistemologia do pensamento sistêmico oferece pressupostos ao pesquisador que possibilitam o estudo de fenômenos considerando a complexidade dos mesmos e a intersubjetividade implicada no estudo. Contudo, os pesquisadores ainda encontram dificuldades referentes aos métodos de coleta de dados e principalmente, como relacionar as diferentes variáveis a serem estudadas, sem que o relatório científi- co se torne pouco atraente e que seus resultados contribuam de fato para o desenvolvimento científico na área.

Pensar a família então, implica em considerar que o comportamento de cada um de seus membros é interdependente do comportamento dos outros, assim, o grupo familiar é visto como um conjunto, como uma totalidade cuja particularidade de um de seus membros não basta para explicar o comportamento dos demais, de forma que a análise de uma família não é a soma da análise de seus membros individuais. Nesse sentido, a unidade familiar é um sistema composto por indivíduos que podem também ser considerados sistemas por si só e, ao mesmo tempo, uma parte de um sistema, ou seja, um subsistema. Essa unidade familiar também faz parte de um sistema familiar maior que se inclui em outros sistemas mais amplos, como o sistema sociocultural e assim por diante (Cerveny, 2000).

Assim, o pensamento sistêmico sugere que diante da diversidade de aspectos que envolvem o fenômeno família, é fundamental levar em conta a complexidade, através da contextualização, social, histórica e cultural. Nesse sentido, a abordagem teórica fundamentada na epistemologia sistêmica se mostra bastante adequada no desenvolvimento de pesquisas com famílias.

São várias as teorias sistêmicas que podem contribuir para o embasamento teórico dos estudos com famílias, dentre elas: a compreensão do ciclo de vida familiar (Carter \& McGoldrick, 2001), e a teoria Bioecológica do Desenvolvimento Humano (Bronfenbrenner, 1999; Bronfenbrenner \& Morris, 1998).

Carter e McGoldrick (2001) propuseram uma classificação de estágios do ciclo de vida familiar a partir da idéia de que a família apresenta um processo central a ser negociado com relação à expansão, contração e realinhamento do sistema de relacionamentos, para suportar a entrada, saída e o desenvolvimento de seus membros da família. Segundo essas autoras, os estágios do ciclo de vida familiar são: (1) estágio em que os jovens solteiros saem de casa; (2) a união de famílias no casamento: o novo casal; (3) famílias com filhos pequenos; (4) famílias com adolescentes; (5) "lançando os filhos e seguindo em frente" e (6) famílias no estágio tardio da vida. As autoras fazem sugestões a respeito do processo de mudança 
necessário, em cada estágio e nos momentos de transições, que podem ser entendidas como "tarefas" a serem desempenhadas pela família como um todo e por seus membros individualmente.

Independente da temática a ser estudada é muito importante que seja feita uma caracterização das famílias participantes incluindo o momento do ciclo vital em que elas se encontram, isso porque, em função destes estágios, as famílias diferem muito entre si. E ainda, deve-se fazer uma adequação dos participantes, em função do estágio do ciclo vital, aos objetivos do estudo.

Historicamente, a implementação de pesquisas empíricas buscando a compreensão do indivíduo no contexto "família" ocorreu basicamente após a publicação dos trabalhos de Urie Bronfrenbrenner, na década de 1970 (Dessen \& Silva Neto, 2000). Este autor desenvolveu a Teoria Bioecológica do Desenvolvimento Humano, segundo esta teoria, o desenvolvimento humano se dá através de processos progressivamente mais complexos de interações recíprocas e ativas, desenvolvendo o organismo humano biopsicológico e as pessoas, objetos e símbolos no meio imediato. Desta forma, o desenvolvimento é compreendido como um fenômeno complexo que se dá através da interação de quatro núcleos principais: os processos proximais (interações que promovem desenvolvimento); as características pessoais; o contexto e o tempo, denominado "Modelo PPCT" (Bronfenbrenner, 1999; Bronfenbrenner \& Morris, 1998).

Os processos proximais dizem respeito às interações recíprocas e ativas com base regular e períodos prolongados no ambiente imediato. Estas interações promovem o desenvolvimento de ambos os participantes e podem produzir efeitos positivos ou negativos no processo de desenvolvimento (Bronfenbrenner, 1999).

A pessoa, na teoria Bioecológica, é entendida como produtora e produto do desenvolvimento, que apresenta características atuantes. O terceiro elemento da teoria, o contexto, é analisado em quatro níveis. $\mathrm{O}$ microssistema, onde a criança vive e interage facea-face seu meio imediato (família-escola), onde ope- ram os processos proximais. No mesossistema ocorrem inter-relações de microsistemas (família-escola). O exossistema diz respeito às inter-relações do(s) microsistema(s) da criança com outro microsistema do qual ela não participa, mas sofre influência (escola-comunidade; família-local de trabalho dos pais). E o macrossistema que se refere aos modelos institucionais de cultura (economia; leis; políticas públicas; religião; costumes) que estabelecem padrões no micro, meso e exossistema.

O quarto elemento da teoria, o tempo, chamado de "cronossistema" abrange as mudanças relacionadas ao tempo no indivíduo e no ambiente em que vive. São as mudanças no ciclo de vida individual e familiar. É dividido em: microtempo (continuidades/descontinuidades dentro dos episódios de processo proximal); mesotempo (periodicidade dos episódios de processo proximal) e macrotempo (transgeracionalidade e mudanças na sociedade).

A teoria Bioecológica requer que um delineamento de pesquisa inclua os seus quatro componentes, demonstrando a interdependência deles. De acordo com Bronfenbrenner, a análise da influência destes aspectos sobre o desenvolvimento humano só é possível quando se emprega um modelo teóricometodológico que permite sua observação (Cecconello \& Koller, 2004).

Esta breve apresentação da teoria Bioecológica permite visualizar sob a perspectiva da complexidade, a compreensão do desenvolvimento humano como um processo que ocorre ao longo de toda a vida. Esta teoria oferece ao pesquisador uma visão ampla das situações, das pessoas e suas inter-relações nos diversos contextos, sendo, portanto, um referencial teórico aconselhável quando se pretende uma compreensão da complexidade do fenômeno família, considerando todos os contextos com os quais ela se relaciona.

Através de revisões críticas de um conjunto de pesquisas, Bronfenbrenner (1986) afirma que a maior parte dos estudos concentrou-se nos processos intrafamiliares de interações diádicas ou triádicas (pai/mãe-filho) e propõe a ampliação do foco através de investigações de como os processos intrafamiliares são afetados pelas condições extrafamiliares. 
A perspectiva sistêmica nos estudos com famílias trouxe implicações da ampliação do foco, mas também de uma mudança de postura e visão das famílias. Muda-se da "visão negativa", na qual o mundo familiar tem como figura principal os desajustes, conflitos, déficits e fracassos (considerando as pessoas nos seus aspectos mais redutores e ainda, muitas vezes, culpabilizando os membros da família a partir de uma lógica de causalidade linear) para focar, pesquisar, compreender e fortalecer, os recursos e o sucesso do grupo familiar, através do estudo de percepções de elementos das experiências de vida, aspectos biológicos, de interações pessoais com o contexto, compreendidos na ótica sistêmica (contextualizada e intersubjetiva).

Dessen e Silva Neto (2000) afirmam que as questões sobre família vêm sendo apontadas como uma das tendências futuras nas pesquisas em psicologia. Citam o exemplo da revista American Psychologist, que dedicou, em janeiro de 2001, a sua seção de "Perspectivas Internacionais" às questões de família. As autoras ressaltam ainda a necessidade de pesquisas na área de família, sob a perspectiva do desenvolvimento humano, que consiste em estudar as fases de desenvolvimento humano considerando o que acontece na família enquanto grupo.

Esta tendência pode ser visualizada nas universidades brasileiras, com os núcleos de pesquisa que se dedicam aos estudos de famílias, e também em livros e periódicos científicos que dedicaram volumes e seções exclusivos a publicações de pesquisas e artigos teóricos sobre famílias.

Zamberlan e Biasoli-Alves, em 1997, publicaram o livro Interações familiares: Teoria, pesquisa e subsídios à intervenção, que reúne diversas pesquisas com famílias brasileiras e apresenta também sugestões de como investigar e intervir socialmente nesses contextos. As pesquisas apresentadas nesta obra abordam, sobretudo, a temática dos fatores de risco em famílias de baixa renda e programas preventivos e promotores do desenvolvimento familiar.

A revista Psicologia em Estudo (Boarini, 2003) publicou em número especial apenas artigos sobre famílias abordando diversas temáticas: casamento e família no século XXI; arranjos familiares de crianças em camadas populares; rede de apoio social e afetiva em situação de doença crônica degenerativa na família; papel da mulher no contexto familiar; práticas educativas, estilos parentais e abuso físico no contexto familiar; representação de jovens sobre os avós; resiliência individual e familiar.

A revista Psicologia: Teoria e Pesquisa (2000) publicou a seção especial "Questões de família e desenvolvimento" em três números (Silva Neto, 2000a; Silva Neto, 2000b; Silva Neto, 2000c), em que podem ser encontradas pesquisas e artigos teóricos que abordam as seguintes temáticas: envolvimento parental; a criança e a família; relação pais e filhos; papéis sociais e envelhecimento sob perspectiva do curso de vida; inter-relação entre os subsistemas conjugal e parental; sensibilidade materna; separação conjugal; envolvimento do pai no cuidado de bebês; rede social de apoio nas transições familiares; papel da mulher brasileira no século XX no contexto familiar.

Em termos da produção de grupos de pesquisa dedicados à temática da família encontramos o Núcleo de Família e Comunidade do Programa de Estudos Pós-Graduados em Psicologia Clínica da Pontifícia Universidade Católica de São Paulo (PUC-SP), cujo objeto de pesquisa é a família na realidade brasileira. Este grupo lançou em 2004 a coleção "Família e...", para divulgação dos estudos de docentes e discentes pertencentes ao Núcleo. O primeiro volume da coleção (Cerveny, 2004) aborda as seguintes questões: família e comunicação; família e divórcio; família e doença; família e bioética; família e drogadição; família e resiliência; família e religião; família e mudança; família e deficiência; família e lei. O segundo volume, (Cerveny, 2006) aponta as seguintes temáticas: narrativas; gênero; parentalidade; irmãos; filhos nos divórcios; genealogia; história; estrutura; violência; intervenções sistêmicas com famílias e redes sociais. No ano de 2007, foi lançada outra coleção organizada pela mesma autora, denominada "Família em movimento" (Cerveny, 2007) que foca as transformações das famílias (da família tradicional à família moderna) e questões familiares ligadas à reprodução assistida; migração; meia-idade feminina, dentre outras.

Por sua vez, outro grupo de pesquisa do Laboratório de Psicologia da Saúde, Família e Comunidade (LABSFAC) do Departamento de Psicologia da 
Universidade Federal de Santa Catarina (UFSC) também reúne diversas pesquisas com famílias abordando temas tais como: dependência química e funcionamento familiar (Orth, 2005; Orth \& Moré, 2007); comunicação entre profissionais de saúde e família (Marcon, 2003; Queiroz, Böing, Crepaldi, \& Wendt, 2005); doenças crônicas e hospitalização de crianças (Crepaldi, 1998a, 1998b, 1999; Crepaldi \& Hackbarth, 2002; Crepaldi \& Varella, 2000; Gabarra, 2005; Nieweglowski, 2004; Nieweglowski \& Moré, 2008); comunicação em famílias com crianças e adolescentes com doenças crônicas (Rabuske, 2004); atendimento psicológico a pacientes internados e seus familiares na fase de suspeita de diagnóstico de câncer e/ou aids (Lima, 2003); doença crônica em idosos e cuidadores familiares (Schneider, 2005); dinâmica relacional de casais em estágio tardio (Cardoso, 2006); luto e morte na família (Crepaldi \& Lisboa, 2003; Lisboa, 2002); paternidade (Andreani, 2006; Crepaldi, Andreani, Ristoff, Hammes, \& Abreu, 2006; Motta \& Crepaldi, 2005); rede de apoio familiar (Andreani, Custódio, \& Crepaldi, 2006; Moré, 2005); adoção (Böing \& Crepaldi, 2004; Frassão, 2000); o comportamento de cuidar em famílias em risco psicossocial (Abreu, 2003); famílias com pessoas acometidas por transtorno mental (Pace, 2005); fatores de risco e proteção para o desenvolvimento da criança na transição para a parentalidade (Wendt, 2006); família e escolarização (Andrada, 2007); saúde e desenvolvimento infantil (Molinari, Silva, \& Crepaldi, 2005); atendimento psicológico a famílias na clínica e na comunidade (Crepaldi \& Moré, 2004); a criança na terapia familiar (Crepaldi, 2001); violência intrafamiliar (Barcelos, 2005); relações familiares e desnutrição (Pedro, 2007); impacto da migração no ciclo de vida da família (Moré \& Queiroz, 2007).

\section{A definição do conceito de família}

No desenvolvimento de qualquer pesquisa científica faz-se necessária a elaboração e adoção de conceitos e definições do objeto de estudo, assim, a pesquisa com famílias não pode prescindir desta tarefa. Contudo, entendida como fenômeno complexo e dinâmico, a família assume diferentes configurações, sejam estruturais ou relacionais, que dificultam a elaboração de um conceito único que contemple as diferentes realidades das famílias.
O crescimento do número de pesquisas antropológicas, nas últimas décadas do século $\mathrm{XX}$, mostrou que existe uma enorme diversidade de dinâmicas familiares, de forma que os pesquisadores corroboram a idéia de que não existe um padrão universal de “evolução familiar" (Cerveny, 2000; Coelho, 2005; Fonseca, 2004; Zamberlan \& Biasoli-Alves, 1997).

Biasoli-Alves $(1999,2000)$ aponta evidências de um forte impacto do intenso desenvolvimento tecnológico do século XX sobre a família, acarretando mudanças estruturais, funcionais e de relacionamento. Decréscimo na convivência da família nuclear com a família extensa, sugerindo um distanciamento gradativo dos jovens casais, de suas famílias de origem, em busca de melhores oportunidades de trabalho e a conseqüente necessidade de adaptação e estabelecimento de relações com as vizinhanças e recursos da comunidade. Mudanças com relação ao papel feminino, a perda do prestígio social da função de cuidar da casa e criar os filhos e a crescente inserção da mulher no mercado de trabalho; a redução do índice de natalidade com o advento de métodos anticoncepcionais mais efetivos; aumento do número de famílias chefiadas por mulheres. $\mathrm{O}$ aumento do nível de escolaridade da população vem acompanhado pela escolarização precoce das crianças, passando a escola a preencher as necessidades das crianças de uma família em que os valores estão mudando.

São tantas as variáveis ambientais, sociais, econômicas, culturais, históricas, políticas ou religiosas que determinam as diferentes composições familiares que se mostra impossível a composição de uma definição completa e integradora do que seja família hoje no Brasil. Possíveis definições podem abarcar as dimensões de estrutura familiar (sua composição), as relações estabelecidas entre seus membros e/ou as funções atribuídas às famílias.

Fonseca (2005) ressalta ainda as variações de concepção de família conforme a categoria social. Em camadas médias, a noção de família é mais restrita ao núcleo pais e filhos; nas camadas populares, por sua vez, amplia-se para a rede extensa de parentes. Nesse sentido, ao investigar grupos populares, torna-se fundamental verificar como as atividades do dia-a-dia envolvem uma rede que se estende no espaço para outras casas e até mesmo para outros bairros. 
Traçando as linhas de ajuda mútua, pode-se melhor refletir sobre o que é, nessa instância, a "família" pertinente.

Para cada estudo, deve-se adotar, ressignificar e/ou construir um conceito que seja operativo e que contemple as características das realidades das famílias pesquisadas, e principalmente, que seja compatível ao referencial teórico adotado. Entendidas como fenômeno complexo, com funções multidimensionais, complexas e variadas, as famílias devem ser estudadas a partir de uma perspectiva cultural, histórica e intergeracional. Além disso, é imprescindível que a abordagem teórica e as metodologias adotadas pelo pesquisador sejam amplas para abarcar a complexidade da temática, e os instrumentos apropriados e sensíveis o suficiente para responder às questões em estudo. Sem contar com os atributos que compõem a definição de família, e que se tornam construtos importantes a serem definidos tais como: dinâmica, estrutura, configuração, funcionamento, coesão, diferenciação, harmonia, liderança, dentre outros, que devem ser conceituados antes de avaliados na pesquisa, tendo em vista que dependendo da fundamentação teórico-epistemológica desta, adotar-se-á uma ou outra definição.

\section{Pesquisando famílias: métodos e instrumentos}

Os estudos com famílias podem assumir delineamentos diversos, que serão aqui apontados, incluir métodos considerados úteis nas pesquisas com famílias (propostas que trabalham com a inserção de pesquisadores no campo de investigação), assim como, instrumentos e técnicas de coleta de dados e diferentes formas de análise, sem a pretensão de abarcá-los integralmente.

\section{Inserção ecológica}

Cecconello e Koller (2003, 2004) referem a "Inserção Ecológica", como proposta metodológica para pesquisas com famílias em ambiente natural, com base na Teoria Bioecológica do Desenvolvimento Humano através de um delineamento de pesquisa e análise a partir de seus quatro elementos-chave (PPCT).

Neste método o pesquisador deve fazer parte do contexto. Entende-se que este tem seu próprio processo pessoal de desenvolvimento influenciado pelas interações que estabelece com os participantes da pesquisa. $\mathrm{O}$ pesquisador deve estar disposto a se integrar no contexto, se relacionando de maneira comprometida, ética e mais próxima possível dos participantes (Cecconello \& Koller, 2003, 2004).

Os objetivos da pesquisa devem ser claros para que os dados coletados sejam filtrados e eleitos a fim de responderem a estes. São os objetivos do estudo que dirigem a investigação, focando um fenômeno e um sistema específico. Contudo, para que um estudo adquira validade ecológica, deve considerar a influência das características das pessoas entrevistadas, do contexto no qual estão inseridas, do tempo em que estão vivendo e dos processos proximais ocorridos entre elas e, entre elas e o pesquisador. Assim, entende-se que um estudo tem "validade ecológica" quando, em seu planejamento, estiverem contempladas, o máximo possível, investigações direcionadas a todos os quatro elementos da teoria Bioecológica (Cecconello \& Koller, 2003, 2004).

\section{Pesquisa participante e pesquisa-ação}

A pesquisa participante consiste na participação de pesquisadores/observadores no cotidiano da família. A diferença deste método em comparação à inserção ecológica está no pano de fundo teórico de ambos. Na pesquisa participante, o pesquisador/observador, embora inserido no contexto, não tem o compromisso de estabelecer uma interação próxima com os sujeitos (não estabelece processos proximais) nem o compromisso de permanecer atento aos aspectos das pessoas, suas interações, do tempo e do contexto, que são exigências do método da inserção ecológica, em função da teoria em que está fundamentado.

A pesquisa-ação vai além da simples inserção/ participação do pesquisador no contexto. Este método agrega diversas técnicas de pesquisa social, com as quais se estabelece uma estrutura coletiva, participativa e ativa no nível da captação da informação, requer, portanto, a participação das pessoas envolvidas no problema investigado. Para isso, deve-se levar em consideração como os participantes questionam a situação, como a compreendem e o que querem mudar, despertando neles o desejo da mudança 
e permitindo uma elaboração conjunta dos meios de sua realização (Prati, De Paula Couto, Moura, Poletto, \& Koller, 2008).

\section{Instrumentos e técnicas de coleta de dados}

Os métodos de pesquisa em geral trazem consigo diferentes instrumentos e técnicas de coleta de dados que podem ser utilizados nos estudos com famílias. Além destes, serão apresentados alguns instrumentos e técnicas que não estão diretamente vinculadas a uma metodologia específica, mas que se mostram bastante úteis. São instrumentos que provêm da prática clínica de trabalho com famílias: o genograma familiar, linha de tempo familiar (LTF); ecomapa e mapa de redes.

O genograma, ou genetograma é uma representação gráfica multigeracional da família que pressupõe, na sua elaboração, três níveis: o traçado da estrutura familiar (demografia); o registro informativo da família (dados da sua história); e a representação das relações e funcionamento familiares. Desta forma, o genograma se mostra um instrumento de pesquisa que se destaca por sua praticidade e organização na apresentação dos dados (Castoldi, Lopes, \& Prati, 2006; McGoldrick \& Gerson, 2001; Wendt, 2006).

Cerveny (2000) propõe o uso da Linha do Tempo Familiar junto com o genograma, trata-se de uma linha horizontal em que são registradas as datas e fatos mais importantes da família, permitindo assim, a obtenção de um número maior de informações e uma disposição mais clara das mesmas.

O ecomapa é um instrumento em forma de diagrama que descreve as relações entre a família e a comunidade permitindo a visualização e avaliação das redes de apoio que a família dispõe, tanto afetivas como as sociais, que são os recursos da comunidade e rede institucional de apoio, ou seja, serviços governamentais ou não governamentais disponíveis na comunidade. Nesta representação gráfica tem-se o genograma da família no centro envolto por um círculo, ao redor deste são distribuídas as pessoas ou instituições que compõem a rede, estes, por sua vez, são ligados, por uma linha, a toda a família ou a membros em particular, de acordo com a utilização dos recursos disponíveis (Rocha, Nascimento, \& Lima, 2002).
Na mesma linha do ecomapa, está o mapa de redes, proposto por Sluzki (1997), sendo também utilizado como instrumento de pesquisa, na linha da família e suas redes significativas. Estes instrumentos, que na prática clínica com famílias cumprem função diagnóstica e terapêutica, podem se tornar valiosos instrumentos de coleta de dados nos estudos com famílias. Contudo, sua aplicação enquanto instrumento de pesquisa deve assumir caráter e função diferentes da clínica, o que requer muita precaução e habilidade do pesquisador.

O genograma, em especial, ao ser construído com as famílias, deflagra descobertas que podem ser agradáveis e instigantes, mas também, revelações e acontecimentos de grande impacto emocional (Cerveny, 2000; Wendt \& Crepaldi, 2008). Os autores que tratam do genograma não se preocuparam em descrever as condições em que este deve ser feito, por se tratar de um instrumento utilizado originalmente no setting clínico, que por si só é continente de efeitos de mobilização emocional. Para o uso em pesquisas, este instrumento deve ser adaptado, sobretudo a entrevista que dá origem ao genograma, que é uma entrevista aberta e bastante peculiar.

Para que o efeito mobilizador seja controlado e minimizado, alguns pesquisadores utilizam o genograma apenas em seu primeiro nível, com o objetivo de obter informações demográficas e da estrutura familiar. Para os estudos em que o foco são as relações familiares e que utilizam o instrumento por inteiro, são realizadas entrevistas para a confecção do genograma, de forma a padronizar a aplicação deste instrumento com todas as famílias participantes e garantir uma investigação de cunho informativo, controlando, de certa forma, o envolvimento e impacto emocional da família (Orht, 2005; Pedro, 2007; Wendt, 2006). Para o desenvolvimento desta entrevista, Wendt (2006) apresenta uma adaptação das intervenções a partir do sistema de categorias desenvolvido por Crepaldi (1989) sobre o significado da comunicação: categorias de incentivo à comunicação; categorias de destaque e clarificação de conteúdos e categoria de apoio.

Mesmo com tais cuidados e precauções, é possível que o uso do genograma produza algum nível de 
mobilização emocional na família, de forma que o pesquisador, por questões éticas, deve ser um profissional habilitado ao manejo deste instrumento e a oferecer apoio aos participantes, em qualquer momento do processo de pesquisa.

Outro instrumento valioso em termos de coleta de dados que acompanha à incursão ecológica em campo é o diário de campo. No mesmo, o pesquisador faz eminentemente, descrições do campo de pesquisa, paralelas à utilização de outro instrumento. Essas descrições se ancoram, por um lado, nos objetivos propostos no planejamento inicial da pesquisa, assim como nos aspectos de intercorrências, derivadas do reconhecimento da imprevisibilidade do campo da pesquisa. Por outro lado, no diário de campo são realizadas descrições sistemáticas das impressões do pesquisador, seja a respeito do campo, como do processo da pesquisa. Os dados do diário de campo se constituem em "dados integradores" relevantes para a análise dos outros instrumentos utilizados, pois auxiliam tanto na compreensão, como na melhor contextualização da análise do campo de pesquisa.

\section{Análise dos dados}

Os dados coletados podem ser analisados segundo uma abordagem quantitativa e/ou qualitativa. A análise quantitativa pode ser de duas formas: quantitativa-descritiva e quantitativa-interpretativa, descritas por Biasoli-Alves (1998).

Há uma tendência de quantificação no estudo com famílias através do uso de escalas para avaliar o funcionamento familiar. Dentre estas a FACES IV, tem sido muito utilizada em estudos internacionais (Olson, Gorall, \& Tiesel, 2007) e é a mais recente versão para avaliar a coesão, flexibilidade e comunicação familiar. Tais escalas utilizam-se de análises estatísticas diversificadas, são em grande número e merecem um estudo à parte.

Flick (2004) ressalta como um dos objetivos da pesquisa qualitativa a demonstração da variedade das perspectivas dos participantes sobre o objeto, partindo dos significados subjetivos e sociais a ele relacionados. Assim, a abordagem qualitativa mostra-se bastante adequada aos estudos com família, pois permite apreensão do caráter multidimensional dos fenômenos estudados, bem como captar diferentes significados de experiências vividas, falas e comportamentos interligados ao contexto. Esta apreensão se mostra delimitada pela abordagem teórica do pesquisador, através de uma sistematização baseada na qualidade, sem a pretensão de atingir a representatividade (Biasoli-Alves, 1998; Flick, 2004).

Embora a abordagem qualitativa permita maior flexibilidade, deve ser salientado o rigor implícito que coloca este método como o mais complexo e exigente quanto à elaboração e cuidados do pesquisador, por não negar a subjetividade, mas trabalhar cuidadosamente com ela, contextualizando os fenômenos. Assim, o ato de pesquisar torna-se uma ação intersubjetiva no sentido de que o pesquisador é seu próprio instrumento, seu próprio termômetro e utiliza sua mobilização para pesquisar. Com relação a esta intersubjetividade, Flick (2004) fala da reflexibilidade do pesquisador e da pesquisa, considerando a comunicação do pesquisador com o campo e seus membros como parte explícita da produção de conhecimento que são documentados em diários de pesquisa ou em protocolos de contexto.

Assim, considerando o ato de pesquisar como uma ação intersubjetiva, a dita "neutralidade" do pesquisador, antes associada ao pressuposto da objetividade, da ciência tradicional, passa a ser relacionada à capacidade de o pesquisador promover a participação de todas as pessoas envolvidas com o fenômeno estudado, dando voz às diferentes partes (Moré, 2000; Moré \& Macedo, 2006). Nesse sentido, no estudo com famílias ressalta-se a necessidade da inclusão de diversos informantes, pois uma das grandes dificuldades na avaliação das pesquisas com famílias é a coleta de dados de um único membro, com extensão dos resultados para a família como um todo. Esta perspectiva individual tomada como familiar, prejudica a validade do estudo e a aplicabilidade dos resultados de pesquisa.

Cabe ressaltar ainda que, embora o método quantitativo esteja ligado ao diretamente observável e mensurável e o qualitativo a um nível de inferência do pesquisador, tais métodos não se opõem, mas se complementam na medida em que representam recortes diferentes de expressão da realidade. Esta combinação pode ser de grande valor em uma pesqui- 
sa, compondo a chamada "triangulação metodológica", que é a complementaridade das análises quantitativas e qualitativas e dos dados observacionais e de entrevista (Biasoli-Alves, s.d).

\section{Considerações finais}

Neste artigo, a utilização de uma abordagem teórica fundamentada na epistemologia sistêmica é apontada como adequada ao desenvolvimento de pesquisas com famílias. Isso não significa que seja a única possível, o que se pretende ressaltar é que a perspectiva sistêmica - através dos pressupostos epistemológicos da complexidade, instabilidade e intersubjetividade - permite ampliar a investigação e discussão acerca desta temática.

Com relação aos métodos utilizados nas pesquisas com famílias, como nos demais estudos científicos, é imprescindível que se tenha em mente que não existe uma estratégia de coleta ou de análise de dados que seja "boa", perfeita ou suficiente em si mesma, essas qualidades dependem de sua adequação ao problema de pesquisa e, sobretudo, da seriedade, rigor e competência da atuação do pesquisador (Romanelli \& Biasoli-Alves, 1998).

Os instrumentos, conforme proposto por Bronfenbrenner (1999), devem ser capazes de capturar os segmentos da "realidade" em diferentes níveis. Estudar as interações e relações desenvolvidas entre os diferentes subsistemas familiares; os contextos histórico, social e econômico no qual as famílias estão inseridas e as famílias em diferentes contextos culturais.

Assim, para estudar famílias, os pesquisadores devem estar dispostos a se integrar no contexto, se relacionando da maneira mais comprometida, ética e próxima possível dos participantes (Cecconello $\&$ Koller, 2004). Sobretudo quando o foco da investigação for as relações familiares, há um grande risco de mobilizar emocionalmente a família e, portanto, o pesquisador deve ser um profissional capacitado para lidar com essas situações e deve estar disponível a responder questões e fornecer apoio à família.

Dessen e Silva Neto (2000) afirmam que planejamentos longitudinais e abordagens multimetodológicas são mais adequados para capturar a complexidade da dinâmica familiar. Cabe ressaltar, entretanto, as dificuldades práticas de desenvolvimento de estudos longitudinais frente a grande mobilidade das famílias, que não permanecem por muito tempo residindo em um mesmo local, sobretudo em classes menos favorecidas, dificultando o acompanhamento das mesmas, e também pela dificuldade de manutenção, a longo prazo, de apoio financeiro a um projeto de pesquisa.

Estudar as famílias através da perspectiva sistêmica implica em uma atitude de contextualização das famílias estudadas e o reconhecimento da causalidade recursiva; entender a família em um processo dinâmico; e reconhecer que não há uma "realidade" das famílias a ser descoberta, mas que a produção de conhecimento, como aponta Esteves de Vasconcellos (2003), é uma construção social em espaços consensuais, por diferentes sujeitos/observadores, admitindo assim, múltiplas versões da realidade, em diferentes domínios lingüísticos de explicações.

Embora se proponha, para as pesquisas com famílias, a adoção da epistemologia sistêmica, diferente da ciência tradicional, isso não significa, em absoluto, o abandono de métodos tradicionais, mas a necessidade do uso adequado e combinado de diferentes abordagens e estratégias metodológicas, entendendo que os conhecimentos científicos representam recortes possíveis e necessários frente à complexidade do fenômeno "famílias".

Os estudos científicos com famílias têm, na atualidade, grande relevância para a ciência, não apenas psicológica, mas para diversos campos de conhecimento. As famílias se constituem, portanto, um campo de estudo heterogêneo, dele se ocupando diferentes disciplinas, diferentes visões complementares cuja integração permite a análise de sua complexidade.

Além disso, as pesquisas científicas com famílias assumem um importante papel social, uma vez que diversos setores públicos e privados têm seus trabalhos direcionados às famílias, dentre eles: saúde (as políticas públicas de saúde têm como foco a família através da Estratégia de Saúde da Família), educação, direito e social. A relevância social que se pretende destacar, corroborada pelas afirmações de Coelho (2005), reside, sobretudo, no fato de que os estudos científicos possibilitam a problematização teórica de vários aspectos e situações, permitindo o 
desenvolvimento de uma visão crítica de preconceitos, crenças e representações dos profissionais sobre as famílias.

\section{Referências}

Abreu, S. R. de (2003). O comportamento de cuidar em famílias em situação de risco psicossocial com crianças em cuidados alternativos. Dissertação de mestrado não-publicada, Universidade Federal de Santa Catarina, Florianópolis, SC.

Andrada, E. G. C. (2007). O treinamento de suporte parental (TSP) como fator de promoção do suporte parental e do desempenho escolar de crianças na primeira série. Tese de doutorado não-publicada, Universidade Federal de Santa Catarina, Florianópolis.

Andreani, G. (2006). Satisfação e responsabilidade: $O$ envolvimento do pai na gravidez durante a transição para a parentalidade. Dissertação de mestrado não-publicada, Universidade Federal de Santa Catarina, Florianópolis.

Andreani, G., Custódio, Z. A. O., \& Crepaldi, M. A. (2006). Tecendo as redes de apoio na prematuridade. Aletheia, 24, 115-126.

Aun, J. G., Esteves de Vasconcellos, M. J., \& Coelho, S. V. (2005). Atendimento de famílias e redes sociais: Fundamentos teóricos e epistemológicos. Belo Horizonte: Ophicina da Arte \& Prosa.

Barcelos, W. B. E. (2005). Violência intrafamiliar: Ressonâncias na prática profissional do psicólogo. Dissertação de mestrado não-publicada, Universidade Federal de Santa Catarina, Florianópolis, SC.

Biasoli-Alves, Z. M. M. (1998). A pesquisa em psicologia: Análise de métodos e estratégias na comunicação na construção de um conhecimento que se pretende científico. In G. Romanelli \& Z. M. M. Biasoli-Alves (Orgs.), Diálogos metodológicos sobre a prática de pesquisa (pp. 135-158). Ribeirão Preto, SP: Legis Summa.

Biasoli-Alves, Z. M. M. (1999). Relações familiares. Texto Contexto Enfermagem, 8, 229-241.
Biasoli-Alves, Z. M. M. (2000). Continuidades e rupturas no papel da mulher brasileira no século XX. Psicologia: Teoria e Pesquisa, 16, 233-239.

Biasoli-Alves, Z. M. M. (s.d). Das inserções quantitativo x qualitativo. Manuscrito não-publicado, Universidade de São Paulo, Ribeirão Preto.

Boarini, M. L. (Ed.). (2003). Psicologia em Estudo, 8 (No. especial).

Böing, E., \& Crepaldi, M. A. (2004). Os efeitos do abandono para o envolvimento psicológico de bebês e a maternagem como fator de proteção. Estudos de Psicologia, 21, 211-226.

Bronfenbrenner, U. (1986). Ecology of the family as a context for human development: Research perspectives. Developmental Psychology, 22, 723-742.

Bronfenbrenner, U. (1999). Environments in developmental perspective: Theorical and operational models. In S. L. Friedmann \& T. D. Wacks (Eds.), Measuring environment across the life span: Emerging methods and concepts (pp.330). Washington, DC: American Psychological Association.

Bronfenbrenner, U., \& Morris. P. A. (1998). The ecology of developmental processes. In W. Damon (Series Ed.) \& R. M. Lerner (Vol. Ed.), Handbook of child psychology: Vol. 1. Theoretical models of human development (pp. 993-1027). New York: John Wiley \& Sons.

Cardoso, V. S. (2006). Tudo que eu fiz, não tenho nada que me arrepender: Percepções e vivências de casais de idosos sobre o estágio tardio. Dissertação de mestrado nãopublicada, Universidade Federal de Santa Catarina, Florianópolis.

Carter, B., \& McGoldrick, M. (2001). As mudanças no ciclo de vida familiar. Porto Alegre: Artes Médicas.

Castoldi, L., Lopes, R. C. S., \& Prati, L. E. (2006). O genograma como instrumento de pesquisa do impacto de eventos estressores na transição família-escola. Psicologia Reflexão e Crítica, 19, 292-300. 
Cecchin, G. (1996). Construcionismo social e irreverência terapêutica. In D. F. Schirtman (Org.), Novos paradigmas, cultura e subjetividade (pp. 216-224). Porto Alegre: Artes Médicas.

Cecconello, A. M., \& Koller, S. H. (2003). Inserção ecológica na comunidade: Uma proposta metodológica para o estudo de famílias em situação de risco. Psicologia Reflexão e Crítica, 16, 515-524.

Cecconello, A. M., \& Koller, S. H. (2004). Inserção ecológica na comunidade: Uma proposta metodológica para o estudo de famílias em situação de risco. In S. Koller (Org.), Ecologia do desenvolvimento humano (pp. 267-292). São Paulo: Casa do Psicólogo.

Cerveny, C. M. O. (2000). A família como modelo: Desconstruindo a patologia. Campinas, SP: Livro Pleno.

Cerveny, C. M. O. (Org.). (2004). Família e... . São Paulo: Casa do Psicólogo.

Cerveny, C. M. O. (Org.). (2006). Família e... narrativas, gênero, parentalidade, irmãos, filhos nos divórcios, genealogia, história, estrutura, violência, intervenção sistêmica, rede social. São Paulo: Casa do Psicólogo.

Cerveny, C. M. O. (Org.). (2007). Família e movimento. São Paulo: Casa do Psicólogo.

Coelho, S. V. (2005). Abordagens psicossociais da família. In J. G. Aun, M. J. Esteves de Vasconcellos \& S. V. Coelho (Orgs.), Atendimento de famílias e redes sociais: Fundamentos teóricos e epistemológicos (pp. 145-232). Belo Horizonte: Ophicina da Arte \& Prosa.

Crepaldi, M. A. (1989). Hospitalização infantil: Estudo das interações família/equipe hospitalar. Dissertação de mestrado não-publicada, Pontifícia Universidade Católica do Rio de Janeiro, RJ.

Crepaldi, M. A. (1998a). Famílias de crianças acometidas por doenças crônicas: Representações sociais da doença. Paidéia (Ribeirão Preto), 8, 157-162.
Crepaldi, M. A. (1998b). Famílias de crianças hospitalizadas: Os efeitos da doença e da internação. Revista Ciências da Saúde, 17, 82-92.

Crepaldi, M. A. (1999). Programas de hospitalização conjunta: Integrando os pais em enfermarias pediátricas. Temas de Psicologia, 7, 152-157.

Crepaldi, M. A. (2001). A importância da participação de crianças na terapia familiar. Acatef em Revista, 1(1), 19-20.

Crepaldi, M. A., Andreani, G., Ristoff, C. D., Hammes, P. S., \& Abreu, S. R. de. (2006). A participação do pai nos cuidados da criança, segundo a concepção de mães. Psicologia em Estudo, 11, 579-587.

Crepaldi, M. A., \& Hackbarth, I. D. (2002). Aspectos psicológicos de crianças hospitalizadas em situação pré-cirúrgica. Temas de Psicologia, 10, 99-112.

Crepaldi, M. A., \& Lisboa, M. L. (2003). Ritual de despedida em familiares de pacientes com prognóstico reservado. Paidéia (Ribeirão Preto), 13, 97-109.

Crepaldi, M. A., \& Moré, C. L. O. O. (2004). Atendimento psicológico a famílias na clínica e na comunidade: Questões ético-metodológicas. Temas de Psicologia, 10, 201-209.

Crepaldi, M. A., \& Varella, P. B. (2000). A recepção da família na hospitalização de crianças. Paidéia (Ribeirão Preto), 12, 23-31.

Dessen, M. A., \& Murta, S. G. (1997). A metodologia observacional na pesquisa em psicologia: Uma visão crítica. Cadernos em Psicologia, 1, 47-60.

Dessen, M. A., \& Silva Neto, N. A. (2000). Questões de família e desenvolvimento e a prática de pesquisa. Psicologia: Teoria e Pesquisa, 16, 191-192.

Esteves de Vasconcellos, M. J. (2003). Pensamento sistêmico: O novo paradigma da ciência. Campinas, SP: Papirus.

Esteves de Vasconcellos, M. J. (2005). Pensamento sistêmico novo-paradigmático: Novo-paradigmático, por quê? In J. G. Aun, M. J. Esteves de Vasconcellos \& S. V. Coelho (Orgs.), Atendimento de famílias e redes sociais: Fundamentos teóricos e epistemológicos (pp. 71-142). Belo Horizonte: Ophicina da Arte \& Prosa. 
Flick, U. (2004). Uma introdução à pesquisa qualitativa. Porto Alegre: Bookman.

Fonseca, C. (2004). Olhares antropológicos sobre a família contemporânea. In C. R. Althoff, I. Elsen \& R. G. Nitscke (Orgs.), Pesquisando a família: Olhares contemporâneos (pp. 55-68). Florianópolis: Papa-Livro.

Fonseca, C. (2005). Concepções de família e práticas de intervenção: Uma contribuição antropológica. Saúde e Sociedade, 14(2), 50-59.

Frassão, M. C. (2000). Devolução de crianças colocadas em famílias substitutas: Uma compreensão dos aspectos psicológicos através dos procedimentos legais. Dissertação de mestrado não-publicada, Universidade Federal de Santa Catarina, Florianópolis, SC.

Gabarra, L. M. (2005). Crianças hospitalizadas com doenças crônicas: A compreensão da doença. Dissertação de mestrado não publicada, Universidade Federal de Santa Catarina, Florianópolis, SC.

Goolishian, H. A., \& Anderson, H. (1996). Narrativa e self: Alguns dilemas pós-modernos da psicoterapia. In D. F. Schnitman (Org.), Novos paradigmas, cultura e subjetividade (pp. 191-200). Porto Alegre: Artes Médicas.

Grandesso, M. (2000). Sobre a reconstrução do significado: Uma análise epistemológica $e$ hermenêutica da prática clínica. São Paulo: Casa do Psicólogo.

Lima, S. de (2003). Atendimento psicológico a pacientes internados e seus familiares na fase de suspeita de diagnóstico de Câncer elou AIDS. Dissertação de mestrado não-publicada, Universidade Federal de Santa Catarina, Florianópolis, SC.

Lisboa, M. L. (2002). Dizendo adeus: Efeitos terapêuticos do ritual de despedida na eminência da morte, em familiares e paciente com prognóstico reservado. Dissertação de mestrado não-publicada, Universidade Federal de Santa Catarina, Florianópolis, SC.
Marcon, C. (2003). A consulta pediátrica e os aspectos comunicacionais entre o médico residente, a criança e sua família. Dissertação de mestrado não-publicada, Universidade Federal de Santa Catarina, Florianópolis, SC.

Maturana, H., \& Varela, F. (1995). A árvore do conhecimento. São Paulo: Psy II.

McGoldrick, M., \& Gerson, R. (2001). Genetograma e o ciclo de vida familiar. In B. Carter \& M. McGoldrick (Orgs.), As mudanças no ciclo de vida familiar (pp. 144-164). Porto Alegre: Artes Médicas.

Molinari, J. S. O., Silva, M. F. M. C., \& Crepaldi, M. A. (2005). Saúde e desenvolvimento da criança: A família, os fatores de risco e as ações na atenção básica. Revista Psicologia Argumento, 23(43), 17-26.

Moré, C. L. O. O. (2000). Atendendo à demanda: Proposta de um modelo de sistematização de intervenção psicológica junto a postos de saúde comunitário. Tese de doutorado não-publicada, Pontifícia Universidade Católica de São Paulo, São Paulo, SP.

Moré, C. L. O. O. (2005). As redes pessoais significativas como instrumento de intervenção psicológica. Paidéia (Ribeirão Preto), 15, 267-297.

Moré, C. L. O. O., \& Macedo, R. M. S. (2006). A psicologia na comunidade: Uma proposta de intervenção. São Paulo: Casa do Psicólogo.

Moré, C. L. O. O., \& Queiroz, A. H. (2007). Migração, movimento e transformação: Irrupção do novo nas relações familiares. In C. M. O. Cerveny (Org), Família e movimento (pp. 54-68). São Paulo: Casa do Psicólogo.

Morin, E. (1990). Introdução ao pensamento complexo. Lisboa: Epistemologia e Sociedade.

Morin, E. (1996). Epistemologia da complexidade. In D. F. Schnitman (Org.), Novos paradigmas, cultura e subjetividade (pp. 274-286). Porto Alegre: Artes Médicas.

Motta, C. C. L. da, \& Crepaldi, M. A. (2005). O pai no parto e apoio emocional: A perspectiva da parturiente. Paidéia (Ribeirão Preto), 15, 105-118. 
Nieweglowski, V. H. (2004). Unidade de terapia intensiva pediátrica: Vozes e vivências da família. Dissertação de mestrado não-publicada, Universidade Federal de Santa Catarina, Florianópolis, SC.

Nieweglowski, V. H., \& Moré, C. L. O. O. (2008). Comunicação equipe-família em unidade de terapia intensiva pediátrica: Impacto no processo de hospitalização. Estudos em Psicologia, 25, 111-122.

Olson, D. H., Gorall, D. M., \& Tiesel, J. W. (2007). FACES IV \& the circumplex model: Validation study. Journal of Family Therapy, 22, 144-167.

Orth, A. P. S. (2005). A dependência química e o funcionamento familiar à luz do pensamento sistêmico. Dissertação de mestrado nãopublicada, Universidade Federal de Santa Catarina, Florianópolis, SC.

Orth, A. P. S., \& Moré, C. L. O. O. (2007). O funcionamento familiar com membros que estabelecem uma relação de dependência com substâncias psicoativas. Psicologia Argumento, 52, 24-38.

Pace, S. E. H. (2005). Um futuro incerto: Projetos e expectativas de familiares que convivem com pessoas acometidas por transtorno mental. Dissertação de mestrado não-publicada, Universidade Federal de Santa Catarina, Florianópolis, SC.

Pedro, F. D. L. (2007). Reconhecendo a dinâmica familiar da familia com filhos desnutridos. Dissertação de mestrado não-publicada, Universidade Federal de Santa Catarina, Florianópolis, SC.

Prati, L. E., De Paula Couto, M. C., Moura, A., Poletto, M., \& Koller, S. H. (2008). Revisando a inserção ecológica: Uma proposta de sistematização. Psicologia: Reflexão e Crítica, 22, 160-169.

Queiroz, A. H., Böing, E., Crepaldi, M. A., \& Wendt, N. C. (2005). Reflexões sobre as representações sociais da AIDS e do câncer e as interações entre pacientes, famílias e profissionais de saúde. Revista de Ciências Humanas, 37, 105-119.
Rabuske, M. M. (2004). O processo comunicativo em familias com crianças e adolescentes doentes crônicos. Dissertação de mestrado nãopublicada, Universidade Federal de Santa Catarina, Florianópolis, SC.

Rocha, S. M. M., Nascimento, L. C., \& Lima, R. A. G. (2002). Enfermagem pediátrica e abordagem da família: Subsídios para o ensino de graduação. Revista Latino-Americana de Enfermagem, 10, 709-714.

Romanelli, G., \& Biasoli-Alves, Z. M. M. (Orgs.). (1998). Diálogos metodológicos sobre a prática de pesquisa. Ribeirão Preto, SP: Legis Summa.

Schneider, P. (2005). O enfrentamento do processo de demência de idosos portadores do tipo Alzheimer pelos familiares cuidadores principais. Dissertação de mestrado nãopublicada, Universidade Federal de Santa Catarina, Florianópolis, SC.

Silva Neto, N. A. e (Ed.). (2000a). Psicologia: Teoria e Pesquisa, 16(1).

Silva Neto, N. A. e (Ed.). (2000b). Psicologia: Teoria e Pesquisa, 16(2).

Silva Neto, N. A. e (Ed.). (2000c). Psicologia: Teoria e Pesquisa, 16(3).

Sluzki, C. E. (1997). As redes sociais na prática sistêmica. São Paulo: Casa do Psicólogo.

Sousa Santos, B. (2006). Um discurso sobre as ciências. São Paulo: Cortez.

Wendt, N. C. (2006). Fatores de risco e proteção para o desenvolvimento da criança durante a transição para a parentalidade. Dissertação de mestrado não-publicada, Universidade Federal de Santa Catarina, Florianópolis, SC.

Wendt, N. C., \& Crepaldi, M. A. (2008). A utilização do genograma como instrumento de coleta de dados em pesquisa qualitativa. Psicologia: Reflexão e Crítica, 21, 302-310.

Zamberlan, M. A. T., \& Biasoli-Alves, Z. M. M. (1997). Interações familiares: Teoria, pesquisa e subsídios à intervenção. Londrina: EdUEL. 
Artigo recebido em 21/12/2007.

Aceito para publicação em 26/08/2008.

Endereço para correspondência:

Elisangela Böing. Rua Lauro Linhares, 1785, bloco leste - apto 103. CEP 88036-002. FlorianópolisSC, Brasil. E-mail: zanziboing@hotmail.com

Elisângela Böing é mestranda pelo Programa de Pós-graduação em Psicologia da Universidade Federal de Santa Catarina.

Maria Aparecida Crepaldi é Professor Associado do Centro de Filosofia e Ciências Humanas da Universidade Federal de Santa Catarina.

Carmen Leontina Ojeda Ocampo Moré é Professor Adjunto do Centro de Filosofia e Ciências Humanas da Universidade Federal de Santa Catarina. 